\title{
Nonadiabatic Dynamics of Photoexcited cis-Stilbene \\ Using Ab Initio Multiple Spawning
}

\section{Supporting Information}

Hayley Weir, ${ }^{1,2}$ Monika Williams, ${ }^{1,2}$ Robert M. Parrish, ${ }^{1,2}$ Edward G. Hohenstein, ${ }^{1,2}$ and

$$
\text { Todd J. Martínez }{ }^{1,2^{*}}
$$

${ }^{1}$ Department of Chemistry and The PULSE Institute, Stanford University, Stanford, California 94305, United States

${ }^{2}$ SLAC National Accelerator Laboratory, 2575 Sand Hill Road, Menlo Park, California 94025, United States

Weir, Williams, Parrish, Hohenstein, Martínez - Stilbene Photodynamics SI - Page S1 


\section{Electronic structure validation}

Multi-state complete active space second-order perturbation theory (MS-CASPT2) is an approximation to the electronic Schrödinger equation that includes dynamic and static electron correlation. Although its high computational expense precludes its use in AIMS simulations for modelling cis-stilbene photodynamics, we can use MS-CASPT2 to generate accurate reference energies at critical points on the potential energy surface (PES) - ground and excited state minima and minimum energy conical intersections (MECIs) along the reaction path. These benchmarks provide validation for more approximate and affordable methods that can be applied in the AIMS simulations.

In addition to good agreement of critical points, the PES must also be continuous. This means that active space methods such as state-averaged complete active space self-consistent field (SA-CASSCF(N,M) where $\mathrm{N}$ and $\mathrm{M}$ are the number of electrons and orbitals, respectively) should not experience rapid rotations of orbitals from within the active space with those outside of the active space.

SA-CASSCF is significantly more computationally affordable than CASPT2 due to the absence of dynamic electron correlation in energy calculations and its implementation on GPUs in TERACHEM. ${ }^{1-3}$ The lack of dynamic electron correlation implies that we should aim to have agreement with trends of the PES of higher levels of theory rather than agreement of absolute values (for example, the vertical excitation energies will usually be too high without proper accounting of dynamic correlation effects).

Critical points along the PES of cis-stilbene, comparing SA-2-CASSCF(2,2) and SA-3MS-CASPT2 $(2,2)$ are shown in Figure S1. The AIMS calculation begins in the excited state at the Franck-Condon (FC) point ( $\mathrm{S}_{0}$ cis-stilbene minimum). From here, trajectory basis functions (TBFs) can either proceed along the isomerisation coordinate, towards the trans-stilbene product, or along the cyclisation coordinate, towards the DHP product. Although CASSCF finds DHP-like and cis-stilbene-like minima on $\mathrm{S}_{1}$, we could not locate these with CASPT2. It is unclear at this stage if these minima exist when dynamic correlation is included. The CASSCF dynamics does not predict significant trapping in these minima, so we omit them from the CASSCF/CASPT2 energy comparison in Figure S1.

Weir, Williams, Parrish, Hohenstein, Martínez - Stilbene Photodynamics SI - Page S2 
The overall shape of the SA-2-CASSCF(2,2) and SA-3-MS-CASPT2(2,2) PESs are consistent, lending support to our choice of CASSCF for the AIMS dynamics. It can be seen, however, that the slope of the PES from the FC point to the twisted $\mathrm{S}_{1}$ minimum, $\mathrm{S}_{1}(\mathrm{pyr})$, is larger for CASSCF than CASPT2, resulting in a wavepacket with a slightly higher potential energy. This implies that the molecule will have more energy (i.e., be hotter) when it returns to the ground state for CASSCF simulations compared to CASPT2.

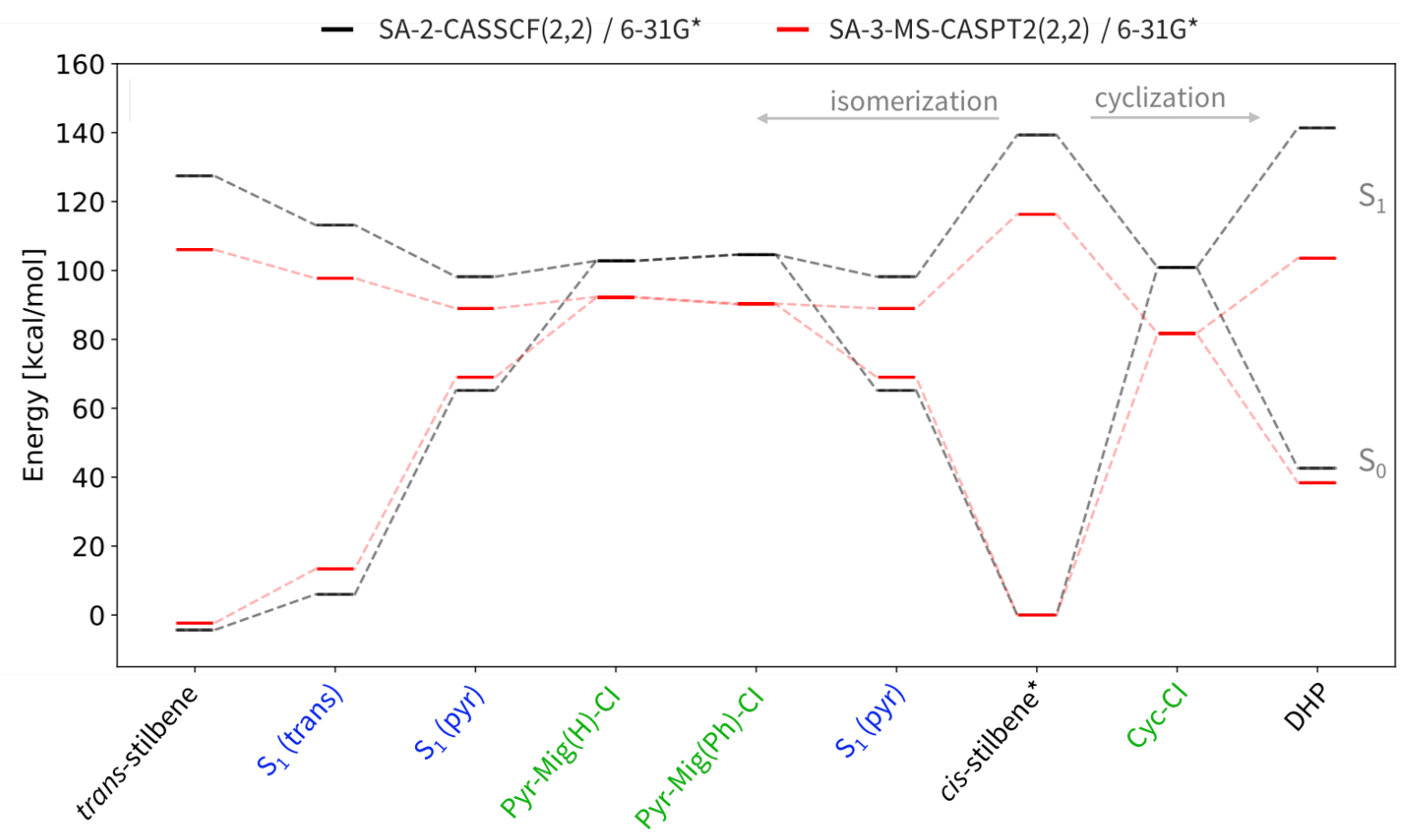

Figure S1. Comparison of CASSCF and MS-CASPT2 at ground $\left(\mathrm{S}_{0}\right)$ and excited $\left(\mathrm{S}_{1}\right)$ state minima and minimum energy conical intersections (MECIs) along the potential energy surface. SA-2CASSCF(2,2)/6-31G* and SA-3-MS-CASPT2(2,2)/6-31G* energies taken from geometry optimizations. Axis labels indicate the type of critical point: ground state minima (black), excited state minima (blue) and $\mathrm{S}_{0} / \mathrm{S}_{1}$ MECIs (green). Cis-stilbene and DHP-like excited state minima were also found with SA-2-CASSCF $(2,2)$ but are not shown. The zero of energy is taken to be the cis-stilbene ground state energy for each electronic structure method.

Weir, Williams, Parrish, Hohenstein, Martínez - Stilbene Photodynamics SI - Page S3 
Table S1. Tabulated energies of ground and excited state critical points along the potential energy surface in $\mathrm{kcal} / \mathrm{mol}$. SA-2-CASSCF(2,2)/6-31G* and SA-3-MS-CASPT2(2,2)/6-31G* energies taken from geometry optimizations. The zero of energy is taken to be the cis-stilbene ground state minimum as computed by the electronic structure method.

\begin{tabular}{lllll}
\hline \hline \multirow{2}{*}{ Critical Point Geometry } & \multicolumn{2}{l}{ SA-2-CASSCF } & $(\mathbf{2 , 2}) / \mathbf{6 - 3 1 G}$ & \multicolumn{2}{l}{ SA-3-MS-CASPT2(2,2)/6-31G* } \\
\cline { 2 - 5 } & $\mathbf{S}_{\mathbf{0}}$ & $\mathbf{S}_{\mathbf{1}}$ & $\mathbf{S}_{\mathbf{0}}$ & $\mathbf{S}_{\mathbf{1}}$ \\
\hline cis-stilbene & 0.0 & 139.342 & 0.0 & 116.297 \\
trans-stilbene & -4.354 & 127.442 & -2.331 & 106.030 \\
DHP & 42.609 & 141.402 & 38.394 & 103.583 \\
$\mathrm{~S}_{1}$ (trans) & 6.012 & 113.173 & 13.382 & 97.722 \\
$\mathrm{~S}_{1}$ (pyr) & 65.188 & 98.1763 & 69.002 & 88.980 \\
$\mathrm{~S}_{1}($ cis-stilbene like) & 22.579 & 55.567 & - & - \\
S $_{1}$ (DHP like) & 60.187 & 60.187 & - & - \\
Pyr-Mig(H)-CI & 102.796 & 102.796 & 92.125 & 92.325 \\
Pyr-Mig(Ph)-CI & 104.588 & 104.588 & 90.173 & 90.391 \\
Cyc-CI & 100.870 & 100.870 & 81.600 & 81.760 \\
\hline \hline
\end{tabular}

SA-2-CASSCF $(2,2) / 6-31 G^{*}$ SA-3-MS-CASPT2(2,2)/6-31G*
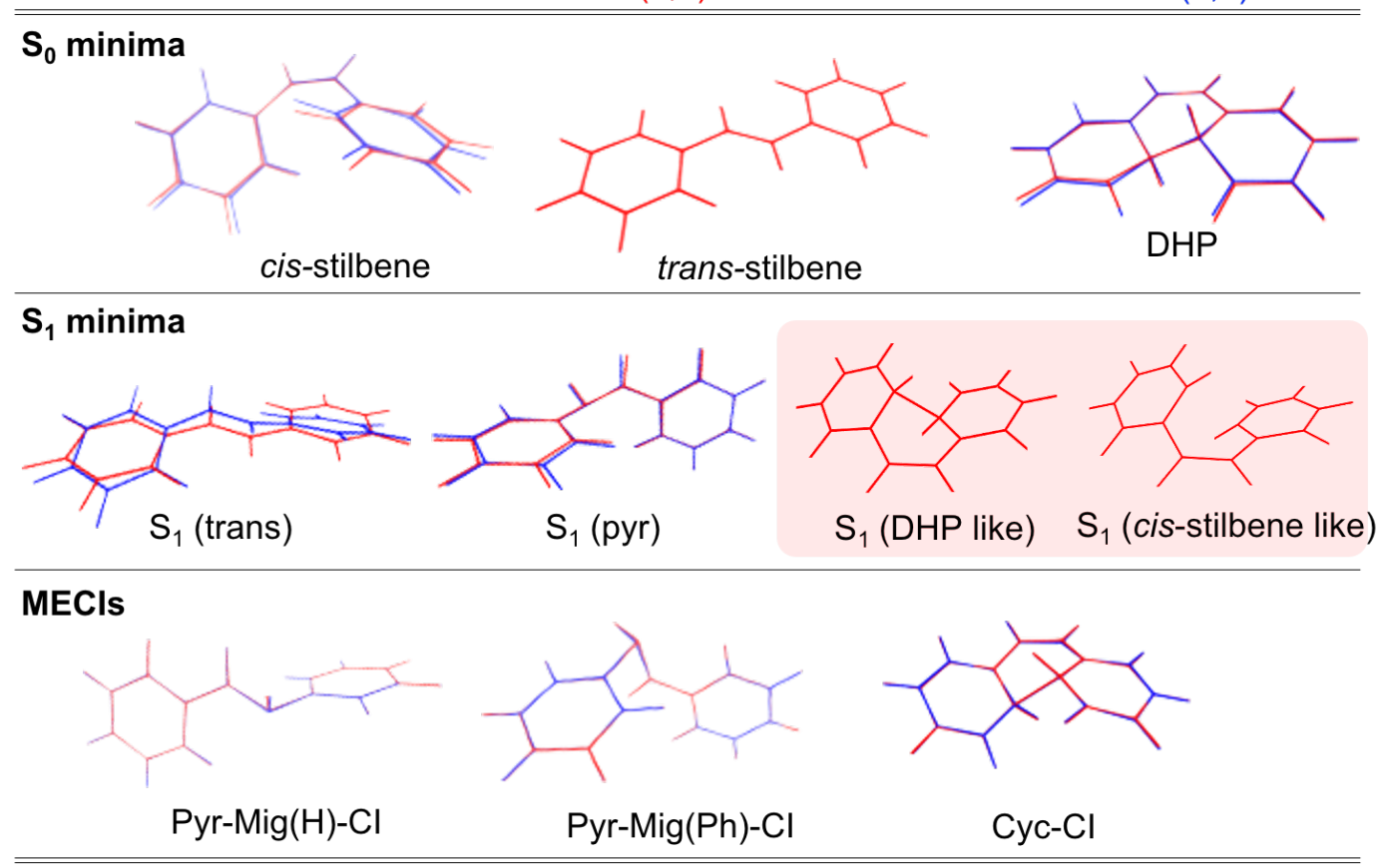

Figure S2. Comparison of geometries of critical points on the potential energy surface calculated with SA-2-CASSCF $(2,2) / 6-31 \mathrm{G}^{*}$ (red) and SA-3-MS-CASPT2(2,2)/6-31G* (blue). S 1 DHP-like and $\mathrm{S}_{1}$ cis-stilbene-like minima (red box) are only found with CASSCF.

Weir, Williams, Parrish, Hohenstein, Martínez - Stilbene Photodynamics SI - Page S4 


\section{Dispersion correction}

A dispersion correction, D3-(BJ), was applied in the optimization and Hessian calculation used in the generation of initial conditions for the dynamics. ${ }^{4}$ The dynamics calculations do not include a dispersion correction (as parameters are not currently available for CASSCF).

\section{Calculation of the photochemical branching ratio}

The photoproducts of AIMS trajectories were measured at 2 ps according to the collective coordinate that we have laid out. By the end of the non-adiabatic simulation, $4.16 \%$ of the wavepacket was not accounted for. This can be attributed to three major reasons, with the percentage of the wavepacket that it accounts for in brackets: (i) trajectories with a population of $<5 \%$ on the ground state after the spawning event are terminated $(0.25 \%)$; (ii) trajectories on $\mathrm{S}_{1}$ that have transferred $>95 \%$ population to the ground state are terminated (1.7\%); (iii) a small number of failed ground state TBFs (1.16\%). The wavepacket was rescaled without bias in order to find the total wavepacket branching ratio. As discussed in the main text, the portion of the wavepacket that resides on the excited state (7\%) at 2 ps is split evenly between cis- and transstilbene.

\section{Example AIMS trajectories}

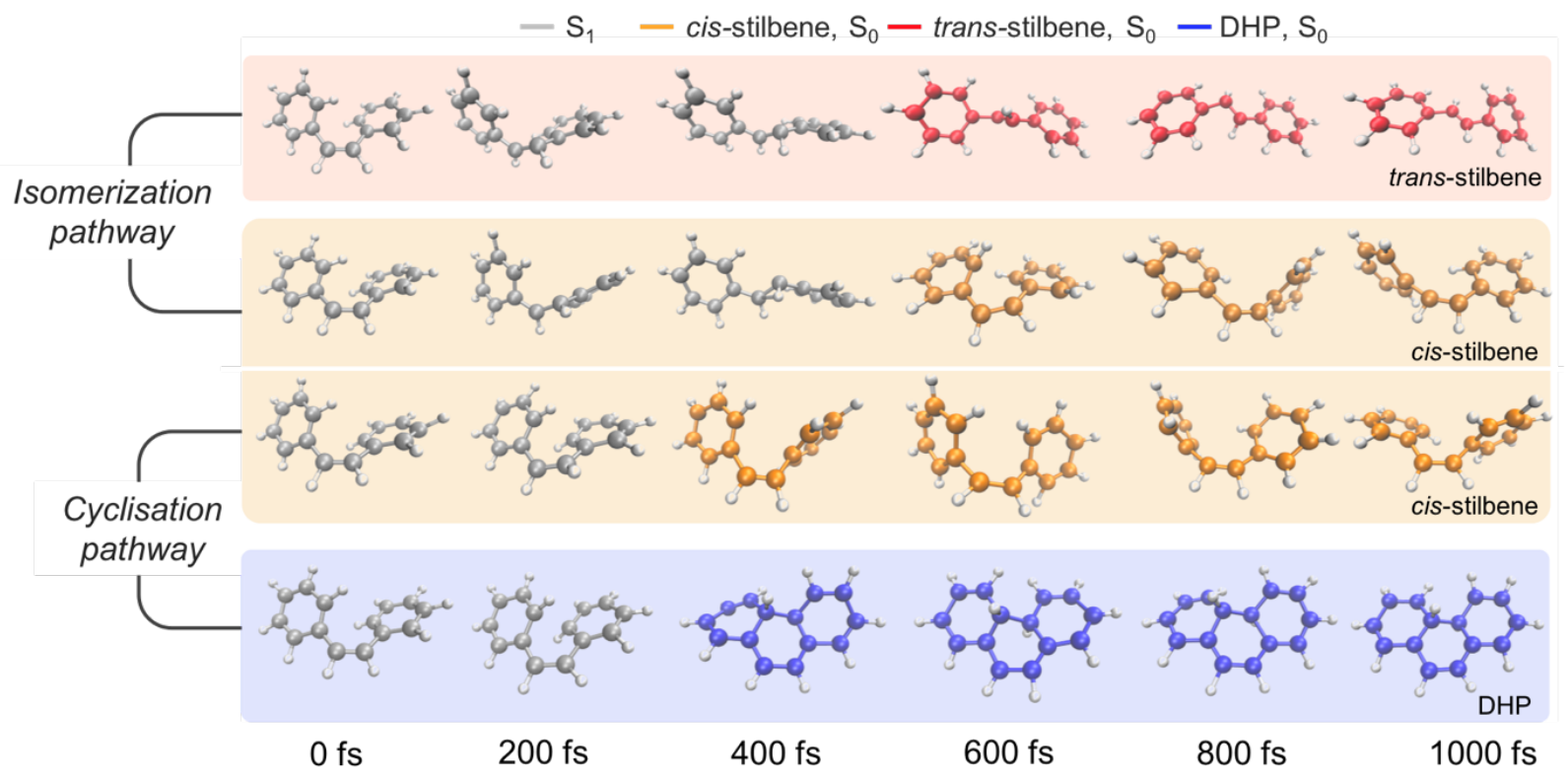

Weir, Williams, Parrish, Hohenstein, Martínez - Stilbene Photodynamics SI - Page S5 
Figure S3. Examples of trajectory basis functions from the AIMS dynamics forming each photoproduct. cis-Stilbene (orange), trans-stilbene (red), DHP (blue) and their reaction coordinate (isomerization vs cyclization) shown.

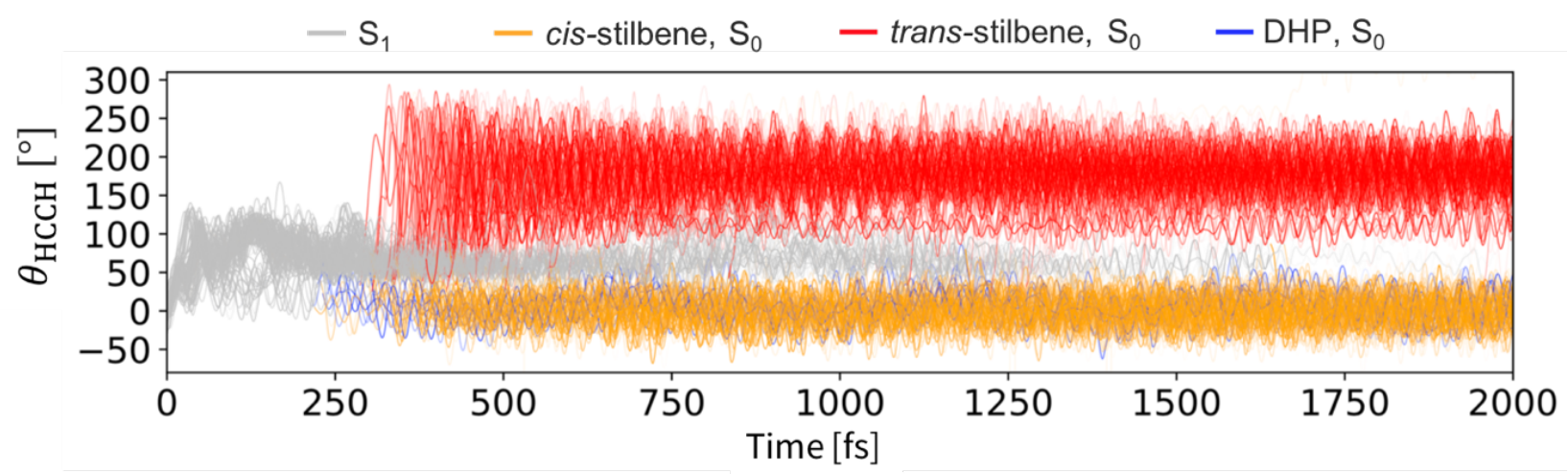

Figure S4. Time evolution of the central ethylenic $\mathrm{H}-\mathrm{C}=\mathrm{C}-\mathrm{H}$ torsion angle, $\theta_{\mathrm{HCCH}}$, along the AIMS trajectory as it decays from $\mathrm{S}_{1}$ to $\mathrm{S}_{0}$ after photoexcitation. The line transparency is proportional to the trajectory weight and colored according to its final product (DHP - blue, cisstilbene - orange, trans-stilbene - red). 


\section{References}

1 Snyder, J. W., Jr.Fales, B. S.Hohenstein, E. G.Levine, B. G. \& Martinez, T. J. A directcompatible formulation of the coupled perturbed complete active space self-consistent field equations on graphical processing units. J. Chem. Phys. 146, 174113, doi:10.1063/1.4979844 (2017).

2 Snyder Jr, J. W.Hohenstein, E. G.Luehr, N. \& Martínez, T. J. An atomic orbital-based formulation of analytical gradients and nonadiabatic coupling vector elements for the state-averaged complete active space self-consistent field method on graphical processing units. J. Chem. Phys. 143, 154107 (2015).

3 Hohenstein, E. G.Luehr, N.Ufimtsev, I. S. \& Martínez, T. J. An atomic orbital-based formulation of the complete active space self-consistent field method on graphical processing units. J. Chem. Phys. 142, 224103 (2015).

4 Grimme, S.Ehrlich, S. \& Goerigk, L. Effect of the damping function in dispersion corrected density functional theory. Journal of computational chemistry 32, 1456-1465 (2011). 